\title{
ISOCAM Deep Surveys and the Cosmic Infrared Background
}

\author{
David Elbaz \\ CEA-Saclay/DAPNIA/Service d'Astrophysique \\ University of California, Santa Cruz: Physics Department $\&$ UCO/Lick \\ Observatory
}

\begin{abstract}
The steep slope of the ISOCAM $15 \mu \mathrm{m}$ number counts indicates that infrared galaxies have strongly evolved since $z \sim 1-1.5$. A nearly complete spectroscopic sample of ISOCAM galaxies in the Hubble Deep Field North (HDFN) shows that their redshift distribution is peaked around $z \sim 0.8$. We show that the $7 \mu \mathrm{m}$ luminosity of local galaxies is correlated with their 8-1000 $\mu \mathrm{m}$ luminosity, and therefore star formation rate (SFR). We use this correlation in the rest-frame of the ISOCAM galaxies to deduce their IR luminosities $\left(\sim 4 \times 10^{11} L_{\odot}\right)$, SFR $\left(\sim 80 M_{\odot} \mathrm{yr}^{-1}\right)$ and contribution to the peak of the cosmic IR background (CIRB) at $140 \mu \mathrm{m}$. We find that they most probably produce the bulk of the CIRB.
\end{abstract}

\section{Introduction: Identification and Nature of the ISOCAM Galaxies}

In Elbaz et al. (1999) we showed that the ISOCAM $15 \mu \mathrm{m}$ differential counts were much steeper than expected if the $15 \mu \mathrm{m}$ luminosity function was to remain identical from $z=0$ to larger redshifts. In the following, we discuss the identification and nature of the sources responsible for this evolution.

The ISOCAM LW3 or $15 \mu \mathrm{m}$ band has a response which covers the range $\lambda=11.5$ to $17 \mu \mathrm{m}$ and is centered at $14.3 \mu \mathrm{m}$. The full width half maximum (FWHM) of the ISO point spread function (PSF) in this band is 4.6 arcsec. In the ultra-deep ISOCAM surveys the final pixel size is $2 \operatorname{arcsec}$ (due to microscanning), which allows some oversampling of the PSF. In the HDFN proper, Pozzetti et al. (1998) measured a density of optical sources with $\mathrm{I}(\mathrm{AB})<29$ of 529 sources per square arcmin ( 2819 objects over an area of 5.33 square arcmin). This corresponds to an average of 9 optical sources per ISOCAM beam. However, the identification of optical counterparts for the ISOCAM $15 \mu \mathrm{m}$ galaxies is strongly facilitated by the facts that:

- above a redshift of $z \sim 1.5$, the rest-frame emission of galaxies drops strongly (the dust emission becomes negligible below $\sim 6 \mu \mathrm{m}$, except for AGNs).

- for the completeness limit of $0.1 \mathrm{mJy}$ reached in the HDFN, only LIRGs (i.e. galaxies with $\left.L(8-1000 \mu \mathrm{m}) \geq 10^{11} L_{\odot}\right)$ are detected above $z=0.67$ and ULIRGs (with $L(8-1000 \mu \mathrm{m}) \geq 10^{12} L_{\odot}$ ) above $z=1.25$, if we assume that the correlation between the mid and far IR emission of galaxies observed in the local universe (see next Section) remains valid at $z \sim 1$. 

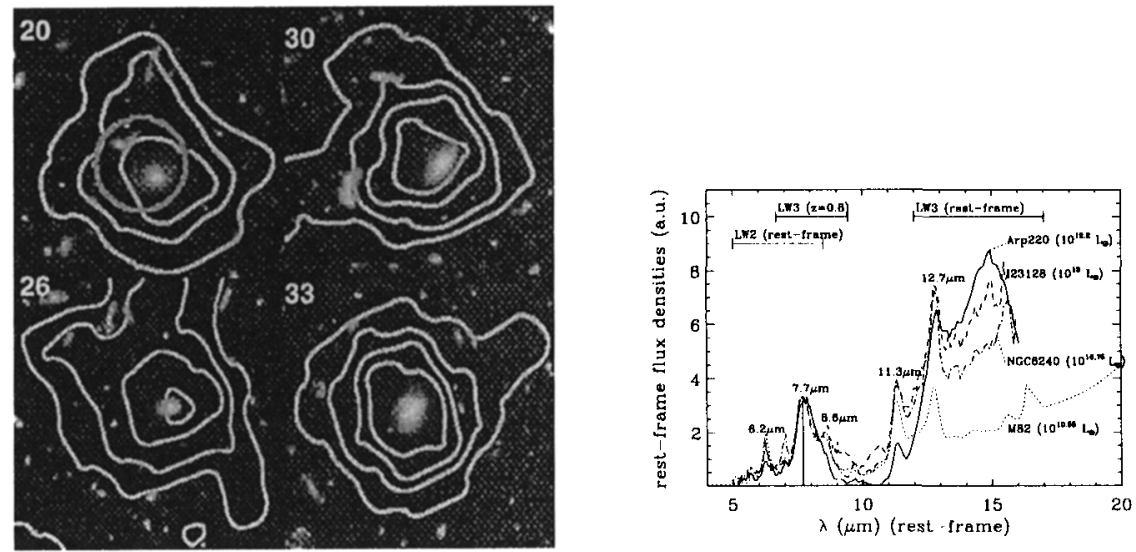

Figure 1. (a): ISOCAM $15 \mu \mathrm{m}$ contour maps of the sources $20(z=$ $0.961), 26(z=0.752), 30(z=0.557), 33(z=0.642)$, overlayed on the optical HDFN image (Aussel et al. 1999). (b): ISOCAM-CVF MIR spectra of 4 galaxies, normalized at $7.7 \mu \mathrm{m}$, as a function of $L(I R)$ (given on the right of the name).

This selection effect explains why the centroid of the isocontours of ISOCAM MIR sources nearly always fall on a relatively bright optical galaxy, as shown by the contour maps on Fig. 1a. This effect is so strong that one can find back the astrometry (better than 2 arcsec) of the ISOCAM contours by matching the pattern of the ISOCAM galaxies with the one of the brightest optical galaxies in the field.

Among a total of 40 galaxies detected by Aussel et al. (1999) in the HDFN + FF above a flux density of $0.1 \mathrm{mJy}$ (completeness limit), 36 have a spectroscopic redshift (8/40 are in the HDFN proper). Their flux densities range from 0.1 to $0.5 \mathrm{mJy}$, i.e. where most of the evolution is observed. At this redshift, the LW3 filter spans the rest-frame $6.2-9.2 \mu \mathrm{m}$ wavelength range which is very close to the ISOCAM LW2 filter $(5-8.5 \mu \mathrm{m}$, centered at $6.75 \mu \mathrm{m})$, hence we can use the correlation that we find between $L(8-1000 \mu \mathrm{m})$ and $L(6.75 \mu \mathrm{m})$ (Fig. 2a) to estimate the IR luminosity and SFR of these galaxies. The results are summarized in Fig. 2b. We find a median IR luminosity of $L(8-1000 \mu \mathrm{m}) \sim 3.6 \times 10^{11} h_{65}^{-2} L_{\odot}\left(\right.$ for $\left.\Omega_{m}=0.3, \Omega_{\Lambda}=0.7\right)$, and assuming that $\mathrm{SFR}=1.7 \times 10^{-10} L(8-1000 \mu \mathrm{m})$ (Kennicutt 1998), we get a median SFR of about $80 M_{\odot} \mathrm{yr}^{-1}$. We separate the contribution of 4 galaxy types as a function of IR luminosity in Table 1.

\section{Cosmic IR Background (CIRB)}

The sum of the contribution of all individual galaxies detected by ISOCAM at $15 \mu \mathrm{m}$ sets a lower limit to the $15 \mu \mathrm{m}$ extragalactic background light (EBL) of $\operatorname{IGL}(15 \mu \mathrm{m})=2.6 \pm 0.5 \mathrm{nWm}^{-2} \mathrm{sr}^{-1}$, down to $0.04 \mathrm{mJy}$. This integrated galaxy light is reduced to $\operatorname{IGL}(>0.1 \mathrm{mJy})=1.6 \pm 0.3 \mathrm{nWm}^{-2} \mathrm{sr}^{-1}$, down to 

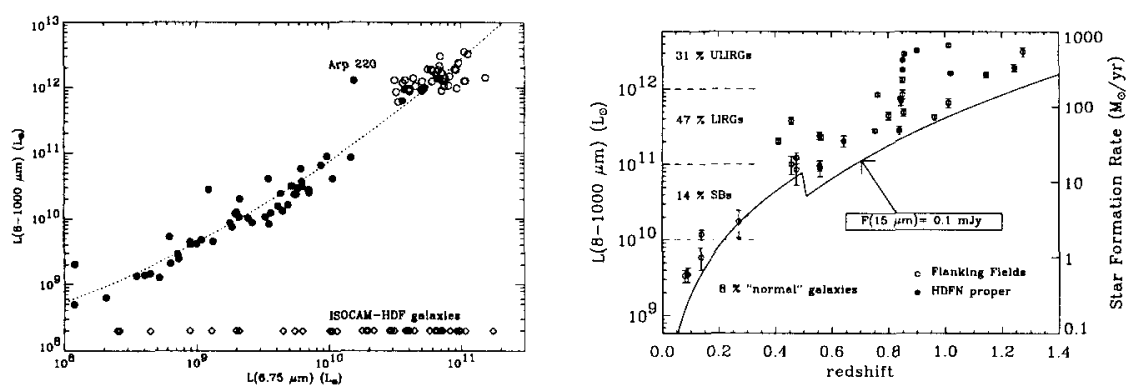

Figure 2. (a): $L(8-1000 \mu \mathrm{m})$ versus $L_{\mathrm{LW} 2}(6.75 \mu \mathrm{m})$. The filled circles come from a sample of local galaxies observed with ISOCAM at LW2 (Chanial et al., in prep.), while the empty circles come from Rigopoulou et al. (1999) ISOPHOT observations at $7.7 \mu \mathrm{m}$ after normalization. The diamonds correspond to the $\mathrm{HDFN}+\mathrm{FF}$ galaxies detected in the LW3 band at $\langle z\rangle \sim 0.8$, i.e. LW2 rest-frame. (b): $L(8-1000 \mu \mathrm{m})$ and SFR of the HDFN $+\mathrm{FF}$ galaxies as a function of redshift (deduced from (a)).

$0.1 \mathrm{mJy}$, if we do not include the small sample of galaxies corrected for lensing magnification by Altieri et al. (1999). More than $80 \%$ of this IGL comes from galaxies fainter than $0.5 \mathrm{mJy}$, typical of the flux densities found in the ISOCAM survey of the HDFN+FF. The bulk of the IGL $(15 \mu \mathrm{m})$ is therefore produced by galaxies at a median redshift of $\langle z\rangle=0.8$, and the wavelength where the CIRB peaks $(140 \mu \mathrm{m})$ corresponds to $80 \mu \mathrm{m}$ in the rest-frame of the ISOCAM galaxies. $L(80 \mu \mathrm{m})$, which is roughly equal to the mean of the IRAS $L(60 \mu \mathrm{m})$ and $L(100 \mu \mathrm{m})$, is almost exactly equal to $L(8-1000 \mu \mathrm{m}) / 2$. One can therefore estimate the $140 \mu \mathrm{m}$ luminosity of the ISOCAM galaxies from the correlation shown in Fig. 1a. The results are shown in Table 1 as a function of the galaxy type (i.e. IR luminosity).

In order to derive these values, we have assumed that the correlation between the far and mid IR luminosities remained valid up to $z=1.3$. We used the $L(\mathrm{IR})$ versus $L(\mathrm{LW} 2)$ correlation for galaxies at $z>0.5$, i.e. whose $7.7 \mu \mathrm{m}$ aromatic feature falls in the LW3 band, and the $L(\mathrm{IR})$ versus $L_{\mathrm{IRAS}}(12 \mu \mathrm{m})$ at $z<0.5$. The ISOCAM detection limit of $0.1 \mathrm{mJy}$ was converted into a $L$ (IR) with these two correlations (plain line in Fig. 2b). The discontinuity around $z=0.5$ is due to the entrance of the $7.7 \mu \mathrm{m}$ major aromatic feature in the LW3 band (see Fig. 1b), i.e. at $z=0.5$ we use a different conversion factor to get $L(\mathrm{IR})$.

We can see that if we use the IGL calculated down to $40 \mu \mathrm{Jy}$, i.e. including lensing magnification, the ISOCAM galaxies produce an IGL at $140 \mu \mathrm{m}$ of $23.4 \pm$ $4.6 \mathrm{nWm}^{-2} \mathrm{sr}^{-1}$, i.e. $94 \%$ of the value measured by DIRBE of $25 \pm 7 \mathrm{nWm}^{-2} \mathrm{sr}^{-1}$ (Finkbeiner, Davis \& Schlegel 2000, Hauser et al. 1998, Lagache et al. 2000). If we only count galaxies above $0.1 \mathrm{mJy}$ (no lensing), then the $\operatorname{IGL}_{\mathrm{CAM}}(140 \mu \mathrm{m})=$ $15.1 \pm 4.6 \mathrm{nWm}^{-2} \mathrm{sr}^{-1}\left(60 \%\right.$ of $\left.\operatorname{EBL}_{\text {DIRBE }}(140 \mu \mathrm{m})\right)$.

In order to use these correlations, we have assumed that the IR light radiated by ISOCAM galaxies was due to star formation and not accretion around 
Table 1. Contribution of the ISOCAM $15 \mu \mathrm{m}$ galaxies to the peak of the CIRB at $140 \mu \mathrm{m}$. Col. $2 \& 3$ are the median redshift and $15 \mu \mathrm{m}$ luminosity. Col. $4 \& 5$ are deduced from the correlation shown in Fig. 2 (right) assuming that the LW3-15 $\mu \mathrm{m}$ luminosity corresponds to the rest-frame LW2-6.75 $\mu \mathrm{m}$ luminosity $(\langle z\rangle=0.8)$. Col. 6 is the fraction of IGL produced by a given galaxy type and Col. 7 is the contribution of these galaxies to the $\operatorname{EBL}(140 \mu \mathrm{m})$, i.e. $\operatorname{IGL}(140 \mu \mathrm{m})$.

\begin{tabular}{|l|l|l|l|l|l|l|}
\hline Type & $\langle z\rangle$ & $\begin{array}{l}\left\langle L_{15 \mu m}\right\rangle \\
\left(L_{\odot}\right)\end{array}$ & $\begin{array}{l}\left\langle L_{I R}\right\rangle \\
\left(L_{\odot}\right)\end{array}$ & $\begin{array}{l}\langle S F R\rangle \\
\left(M_{\odot} / y r\right)\end{array}$ & $\%$ IGL & $\begin{array}{l}\text { IGL }^{C A M}(140 \mu m) \\
n W m^{-2} s r^{-1}\end{array}$ \\
\hline ULIRGs & 1.0 & $9.3 \times 10^{10}$ & $2.4 \times 10^{12}$ & 410 & 40 & $13.4 \pm 2.6$ \\
LIRGs & 0.8 & $2.5 \times 10^{10}$ & $3.8 \times 10^{11}$ & 64 & 42 & $7.7 \pm 1.5$ \\
SBs & 0.5 & $6.2 \times 10^{9}$ & $8.5 \times 10^{10}$ & 14 & 10 & $1.5 \pm 0.3$ \\
normal & 0.1 & $3.0 \times 10^{8}$ & $3.5 \times 10^{9}$ & 0.6 & 8 & $1.3 \pm 0.2$ \\
\hline Total & 0.8 & $3.3 \times 10^{10}$ & $4.5 \times 10^{11}$ & 76 & 100 & $23.9 \pm 4.6$ \\
\hline
\end{tabular}

a black hole. AGNs present flatter spectra, hence their FIR over MIR ratio is smaller, so the larger the AGN contribution, the smaller the $\operatorname{IGL}_{\mathrm{CAM}}(140 \mu \mathrm{m})$ over $\operatorname{IGL}_{\text {CAM }}(15 \mu \mathrm{m})$. A detailed study of the nature of the ISOCAM galaxies is still going on. However, we can already say among the 8 ISOCAM sources brigther than $0.1 \mathrm{mJy}$ detected in the HDFN proper, 2 have been detected in the hard X-ray by Chandra (Hornschemeier et al. 2000), one of which is a spiral galaxy at $z=0.089$ (whose X-ray emission may be due to a SN remnant). The other one is clearly identified as an AGN, but was also clearly stated as such from the optical. It has also been detected in the LW2 band which implies a flat LW3/LW2 ratio typical of AGNs. This is the only galaxy detected both in LW3 and LW2 in the HDFN proper. If the fraction of AGNs is confirmed to be low among the ISOCAM galaxies then our IGL estimate is realistic. Then ISOCAM galaxies most probably produce the bulk of the cosmic IR background and their detailed study is of fundamental importance.

Acknowledgments. This work was supported by NASA grants NAG58218 and NAG5-3507.

\section{References}

Altieri, B., Metcalfe, L., Kneib, J. P., et al. 1999 A\&A, 343, L65

Aussel, H., Cesarsky, C. J., Elbaz, D., Starck, J. L. 1999, A\&A, 342, 313

Elbaz, D., Cesarsky, C.J., Fadda, D., et al. 1999, A\&A, 351, L37

Finkbeiner, D.P., Davis, M., Schlegel, D.J. 2000, ApJ, 524, 867

Hauser, M.G., Arendt, R.G., Kelsall, T., et al. 1998, ApJ, 508, 25

Hornschemeier, A. E., Brandt, W. N., Garmire, G. P., et al. 2000, AJ, 541, 49 Kennicutt, R.C.Jr. 1998, ARA\&A, 36, 189

Lagache, G., Haffner, L.M., Reynolds, R.J., Tufte, S.L. 2000, A\&A, 354, L247

Pozzetti, L., et al. 1998, MNRAS, 298, 1133

Rigopoulou, D., Spoon, H.W.W., Genzel, R., et al. 1999, AJ, 118, 2625 